\title{
Lateral periodontal cyst: a clinicopathological study of 23 cases and an immunohistochemical analysis of its characteristic epithelial plaques in the lining
}

\author{
Masayuki Tsuneki ${ }^{1}$, Jun Cheng ${ }^{1}$, Manabu Yamazaki ${ }^{1}$, Satoshi Maruyama ${ }^{1}$, Takanori Kobayashi ${ }^{2}$, \\ Hiroko Ida-Yonemochi ${ }^{2}$, Makoto Suzuki², Takashi Saku' ${ }^{1,2}$ \\ ${ }^{1}$ Division of Oral Pathology, Niigata University Graduate School of Medical and Dental Sciences, Niigata, Japan \\ ${ }^{2}$ Surgical Pathology Section, Niigata University Hospital, Niigata, Japan
}

Abstract: To characterize lateral periodontal cyst (LPC) clinicopathologically, we have analyzed 23 cases of LPC filed in Niigata University Hospital in the last 13 years. They accounted $1.4 \%$ of jaw cysts $(1,683$ cases total). The mean age of the patients was 46 years, and there were 13 male (56.5\%) and 10 female (43.5\%) patients. Maxillary LPCs were found in the incisor region, and mandibular ones were in the molar region. The maxillary cysts had larger size $(19.8 \mathrm{~mm}$, average diameter) and more frequent association with tooth roots $(80 \%)$ than mandibular cysts $(13.4 \mathrm{~mm}, 20 \%$, respectively). Histopathologically, the linings of LPC were non-keratinized stratified squamous epithelia with occasional cuboidal changes containing small numbers of mucous or sebaceous cells. The base line of the lining epithelium was flat without rete processes. There were characteristic epithelial plaques $(0.5 \mathrm{~mm}$ mean wide and $0.13 \mathrm{~mm}$ high $)$ in all of the cases examined, and round or irregular-shaped epithelial islands were occasionally found in the cyst wall (34.8\%). Immunohistochemically, there were scarcely any Ki-67-positive (+) cells in the lining epithelia, indicating that the lining cells were not proliferative. Both upper surface cells and basal cells of the epithelial plaques were CK19+ and integrin $\beta 1+$, indicating that the epithelial plaques represented certain wrinkle or wavy protrusions of the inner surface of the cyst wall. The histopathological diagnosis of LPC must be precisely done from now on such clinicopathological characteristics are well recognized in this study.

[Oral Med Pathol 2008; 12: 89-96 doi: 10.3353/omp.12.89]

Key words: clinicopathological characteristics, differential diagnosis, epithelial plaque, immunohistochemistry, lateral periodontal cyst

Correspondence: Jun Cheng, Division of Oral Pathology, Department of Tissue Regeneration and Reconstruction, Niigata University Graduate School of Medical and Dental Sciences, 2-5274 Gakkocho-dori, Chuo-ku, Niigata 951-8514, Japan Phone: +81-25-227-2834, Fax: +81-25-227-0805, E-mail: jun@dent.niigata-u.ac.jp

\section{Introduction}

Lateral periodontal cyst (LPC), a developmental odontogenic cyst, was first reported by Mezrow in 1950 (1). Since then, there have been several reports characterizing its clinicopathological features (1-11). LPC occurs in the lateral periodontal region of vital tooth roots mainly around canines, premolars and molars of the mandible. It is usually asymptomatic and is mostly found on routine radiological examinations for purposes other than diagnosing LPC in middle-aged patients (1-11). Radiographically, LPC usually appears round-shaped, with a well-circumscribed radiolucency between tooth roots (1-10). From clinical and radiological findings, it is sometimes difficult to distinguish between LPC and keratocystic odontogenic tumor. Histopathologically, LPC has been characterized by its thin and non-keratinized lining epithelium with focal thickenings referred to as epithelial plaques (2-11). However, LPCs are not always removed in their intact conditions but are usually associated with inflammation, particularly when LPCs have grown into larger sizes. On such occasions, the differential diagnosis of LPC from other jaw cysts becomes difficult. In addition, the LPC lining epithelia, including the characteristic epithelial plaques, have not been well characterized in terms of their proliferating activities and histopathogenesis.

The purpose of this study was to analyze LPC cases documented in our hospital clinicopathologically and immunohistochemically more precisely to differentiate LPC 
lining epithelia from those in other cystic jaw lesions including keratocystic odontogenic tumor, as well as to determine a possible histogenetic process of the epithelial plaques.

\section{Material and Methods}

\section{Cases}

To determine typical histopathological features of LPC, 161 cystic lesions, which were originally diagnosed as primordial cysts or just as cysts, were selected from the surgical pathology files of the Division of Oral Pathology, Niigata University Graduate School of Medical and Dental Sciences, during a thirteen-year period from 1995 to 2007. Their tissue sections stained with hematoxylin and eosin were screened according to the 1992 WHO histopathological criteria of LPC (12) in reference to their clinical data. Clinical data, such as patients' age and sex, locations, sizes, luminal contents, tooth association, bone resorption, clinical symptoms, and clinical diagnoses of LPCs, were also examined. At the same time, ten surgical specimens from each keratocystic odontogenic tumor and radicular cyst were collected for comparison with LPC. The samples, fixed in $10 \%$ formalin and routinely embedded in paraffin, were cut into $4 \mu \mathrm{m}$ sections consecutively and used for immunohistochemical stainings.

\section{Histopathology}

Histological sections of the confirmed LPC cases were reviewed again, and their histopathological characteristics were analyzed in terms of their average thickness of the cyst walls, inflammation, lining epithelial cells of the cystic space, epithelial plaques, epithelial islands, and metaplastic conditions of the epithelial component.

\section{Epithelial plaques}

The sizes of the epithelial plaques were measured, and their immunohistochemical phenotypes were determined.

\section{Antibodies}

Mouse monoclonal antibodies against human keratin 10 (CK10, clone DE-K10, isotype $\operatorname{IgG}_{1} \varkappa$ ), CK17 (E3, $\operatorname{IgG}_{2 b}$ ), CK19 (RCK108, IgG 1 ), Ki-67 (MIB-1, IgG 1 ), and podoplanin (D2-40, $\mathrm{IgG}_{1}$ ) were purchased from Dako (Glostrup, Denmark). A mouse monoclonal antibody against human integrin $\beta 1$ (4B7R, $\mathrm{IgG}_{1}$ ) was purchased from Santa Cruz Biotechnology, Inc. (Santa Cruz, CA, USA).

\section{Immunohistochemistry}

Immunohistochemical staining was performed by using the ChemMate Envision system (Dako). For CK10 and CK17, sections were autoclaved in $10 \mathrm{mM}$ Tris buffer $(\mathrm{pH}$ 9.0) containing $1 \mathrm{mM}$ EDTA at $121^{\circ} \mathrm{C}$ for $10 \mathrm{~min}$. For CK19 and integrin $\beta 1$, sections were pre-treated with $0.2 \%$ trypsin (type II, Sigma Chemical Co, St. Louis, MO, USA) in 0.01 $\mathrm{M}$ Tris- $\mathrm{HCl}(\mathrm{pH} 7.6)$ containing $0.1 \% \mathrm{CaCl}_{2}$ in $30 \mathrm{~min}$ at $37^{\circ} \mathrm{C}$. For Ki-67, sections were autoclaved in citric acid buffer (pH 6.0) at $121^{\circ} \mathrm{C}$ for $10 \mathrm{~min}$. After pre-treatment, the sections were rinsed in $0.01 \mathrm{M}$ phosphate buffered saline (PBS, pH 7.4) containing 0.5\% skimmed milk and $0.05 \%$ Triton X-100 (T-PBS) and treated with $0.3 \%$ hydrogen peroxide in methanol for $30 \mathrm{~min}$ at room temperature to block endogenous peroxidase activities. After rinsing in T-PBS, they were incubated with $5 \%$ skimmed milk in T-PBS for $1 \mathrm{~h}$ at room temperature to block non-specific protein binding sites. They were then incubated over night at $4^{\circ} \mathrm{C}$ with the primary antibodies diluted at 1:100 (DE-K10 and RCK108), 1:40 (E3), 1:50 (4B7R and MIB-1), and 1:200 (D2-40) in T-PBS. After incubation, the sections were rinsed in T-PBS and incubated with the secondary antibodies conjugated with peroxidase-dextran polymers for $1 \mathrm{~h}$ at room temperature. After rinsing with T-PBS, they were treated with $0.02 \%$ 3,3'-diaminobenzimine in $0.05 \mathrm{M}$ Tris$\mathrm{HCl}$ buffer ( $\mathrm{pH} 7.6$ ) containing $0.005 \%$ hydrogen peroxide to visualize the reaction products. Finally, the sections were counterstained with hematoxylin. For control studies on antibodies, the primary antibodies were replaced with preimmune rabbit IgG or mouse IgG subclasses (Dako).

\section{Results}

\section{Frequency}

The diagnosis of lateral periodontal cyst (LPC) was finally confirmed in 23 out of the 161 candidate cases, and they accounted $1.4 \%$ of 1,683 , a total number of cystic jaw lesion cases documented in the same period.

\section{Age and sex}

The age and sex distribution in the 23 cases of LPC is shown in Fig. 1. The mean age of the patients was 46 years

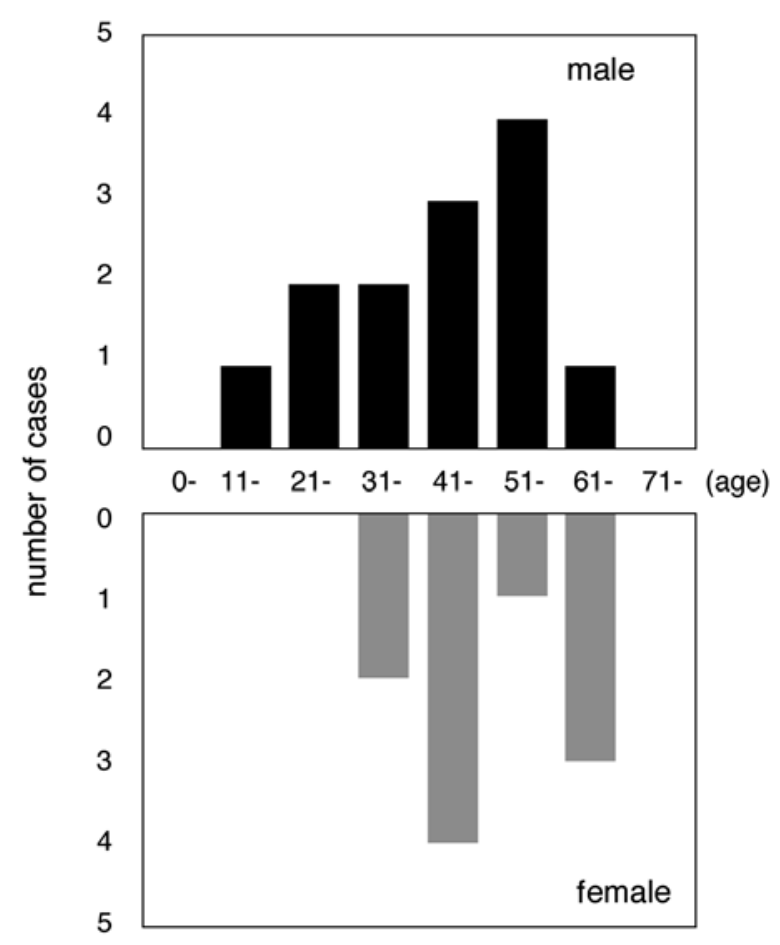

Fig. 1. Age and sex distribution of patients with lateral periodontal cysts from Niigata University Hospital, 1995-2007. Black bar, male; gray bar, female. 
Table 1. Anatomical distribution of lateral periodontal cysts

\begin{tabular}{ccccc}
\hline & \multicolumn{4}{c}{ teeth associated with lateral periodontal cysts } \\
\cline { 2 - 5 } & incisor (\%) & premolar (\%) & molar $(\%)$ & subtotal (\%) \\
\hline $\begin{array}{c}\text { maxilla } \\
\text { left }\end{array}$ & $4(36.4)$ & 0 & $1(100.0)$ & $5(41.7)$ \\
right & $7(63.6)$ & 0 & 0 & $7(58.3)$ \\
\hline subtotal (\%) & $11(100.0)$ & 0 & $1(100.0)$ & $12(100.0)$ \\
\hline mandible & & & & \\
left & 0 & $4(80.0)$ & $3(50.0)$ & $7(63.6)$ \\
right & 0 & $1(20.0)$ & $3(50.0)$ & $4(36.4)$ \\
\hline subtotal $(\%)$ & 0 & $5(100.0)$ & $6(100.0)$ & $11(100.0)$ \\
\hline total $(\%)$ & $11(47.8)$ & $5(21.7)$ & $7(30.4)$ & $23(100.0)$ \\
\hline
\end{tabular}

old ranging the first to sixth decades. There were 13 male $(56.5 \%)$ and 10 female $(43.5 \%)$ patients.

\section{Location}

The anatomical distribution of the 23 LPCs is shown in Table 1. There were 12 maxillary LPCs $(52.2 \%)$ and 11 mandibular cysts $(47.8 \%)$. Twelve cysts occurred in the left side of jaw bones and 11 were in the right side. Eleven cysts involved incisor teeth (47.8\%), 5 premolar teeth $(21.7 \%)$, and 7 molar teeth (30.4\%). LPCs arising in the incisor region were only found in the maxilla, while most of the premolar and molar cases (11/12 cases, $91.7 \%)$ were in the mandible.

\section{Size}

The average long diameter of LPCs was $16.7 \mathrm{~mm}$ ranging from $5 \mathrm{~mm}$ to $35 \mathrm{~mm}$. LPCs of the maxilla were larger in diameter $(19.8 \mathrm{~mm}$, average $)$ than those of the mandible $(13.4 \mathrm{~mm})$. When the maxillary bone was involved, LPCs tended to be expansive between tooth roots forming complicated shapes.

\section{Luminal contents}

The luminal contents of LPCs in surgical records were serous (10 cases, $43.5 \%$ ) or muddy ( 8 cases, $34.8 \%$ ), while no information was obtained even at gross cuttings in the other 5 cases.

\section{Tooth association}

Most of the LPCs were located in the vicinity of tooth roots but were not directly associated with tooth roots. However, tooth roots were involved within the cystic lumina in 5 cases $(21.7 \%)$, most of which (4/5 cases, $80 \%)$ were found in the maxilla. Three out of the 4 maxillary cases had complicated-shaped cystic cavities spread between incisor roots. In contrast, the mandibular cyst was simply located beside the root apex of a molar tooth.

\section{Bone resorption}

Nine cases $(39.1 \%)$ showed cortical bone resorption in their corresponding positions.
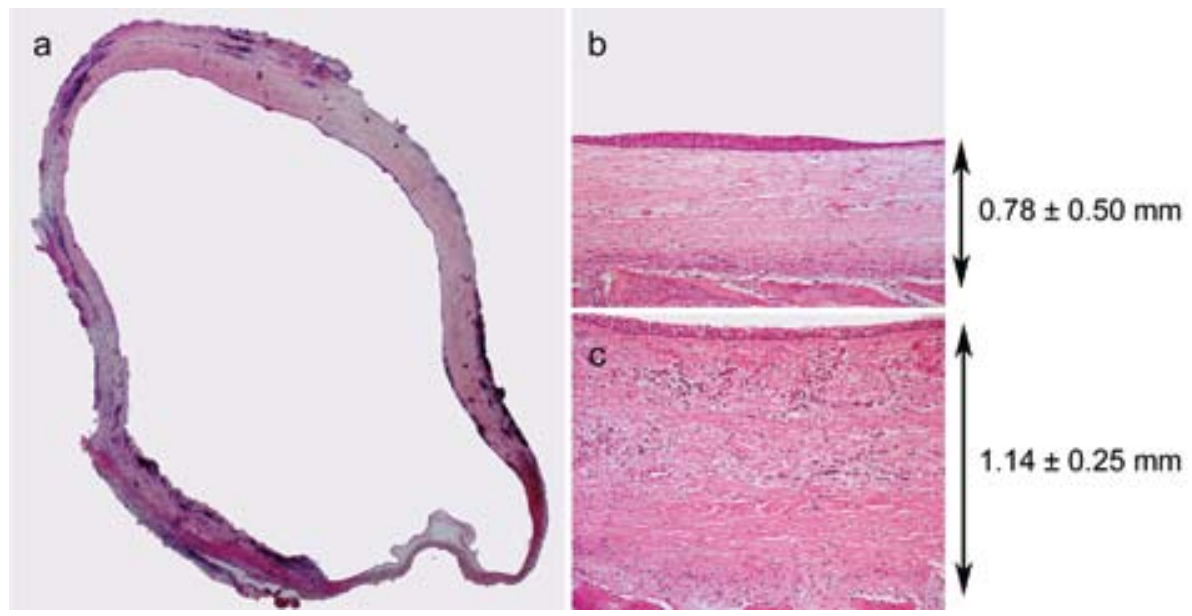

Fig. 2. Histology of lateral periodontal cyst. Hematoxylin and eosin $(\mathrm{HE})$ stain. $(\mathbf{a}): \times 6 ;(\mathbf{b}, \mathbf{c})$ : $\times 25$. (a) Overview of the cyst wall. (b) Cyst walls without inflammation were composed of fibrous connective tissue lined with thin stratified squamous epithelium with socalled epithelial plaque formation, which resulted from focal and sessile protrusion of epithelial cells. Average thickness $0.78 \pm 0.50 \mathrm{~mm}$. (c) Cyst walls with inflammation were composed of thicker fibrous granulation tissue, measuring $1.14 \pm 0.25 \mathrm{~mm}$ in thickness. 


\section{Clinical symptoms}

More than half of the LPCs were found by family dentists of the patients during their dental treatments when $\mathrm{X}$-ray examinations were performed for other purposes (15 cases, $65.2 \%$ ), and the LPCs were asymptomatic. The other 8 cases were noticed by swelling ( 6 cases in maxilla; 2 cases in mandible). The results indicate that LPCs tend to be more expansive in the maxillary bone.

\section{Clinical diagnosis}

Most of the LPCs were diagnosed clinically as cysts not otherwise specified. However, some of them were suspected as keratocystic odontogenic tumor ( 1 case, $4.3 \%$ ), primordial cyst ( 3 cases, 13\%), ameloblastoma (1 case, $4.3 \%$ ), and benign tumor ( 1 case, $4.3 \%$ ). Only one case out of 23 cases (4.3\%) was diagnosed as a LPC according to its typical X-ray finding.

\section{Histopathology}

A loupe view of a cut surface of the cyst wall of LPC is shown in Fig. 2a. The cyst wall was composed of basically thin fibrous connective tissue with an average thickness of $0.78 \pm 0.50 \mathrm{~mm}$ (Fig. 2b). However, it became thicker when it was inflamed and changed into fibrous granulation tissue with an average thickness of $1.14 \pm 0.25 \mathrm{~mm}$ (Fig. 2c). The inner surfaces of LPCs were lined basically by thin stratified squamous epithelium with two to three layers, or sometimes cuboidal epithelial cells (4 cases, $17.4 \%$ ) or pseudostratified ciliated epithelial cells ( 2 cases, $8.7 \%$ ) were contained. In the squamous epithelial lining, there were characteristic changes, so-called epithelial plaques in all of the present series of 23 cases. There were two types of epithelial plaques: exophytic (Fig. 3a, 22 cases, 95.7\%) and invaginated (Fig. 3b, 15 cases, $65.2 \%$ ) types. There was no predilection in the occurrence of epithelial plaques between the maxillary
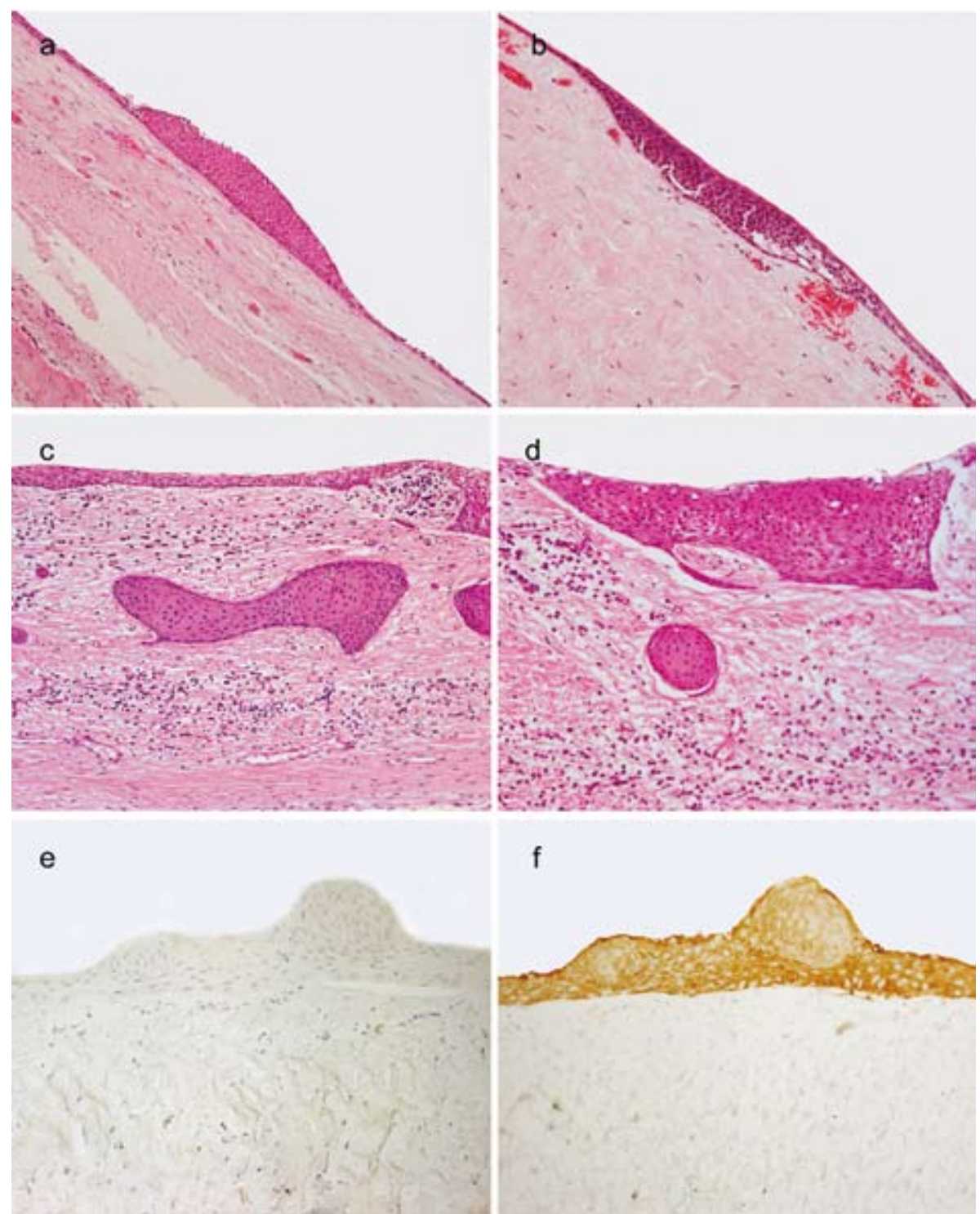

Fig. 3. Characteristic histopathology of lateral periodontal cyst. (a-d) HE stain; (e) immunoperoxidase stain for keratin (CK) 10, hematoxylin counterstain; (f) immunoperoxidase stain for CK17, hematoxylin counterstain. $(\mathrm{a}, \mathrm{b}) \times 60$; $(\mathrm{c}-\mathrm{f}) \times 100$. (a) Typical exophytic epithelial plaque; (b) invaginated epithelial plaque; (c) irregularand (d) round-shaped epithelial islands, maybe a crosscut of parts of invaginated plaques. Immunohistochemically, the lining epithelium of LPC was not positive for CK10 (e), CK17 was demonstrated in the whole layer (f). 
(12 cases, $52.2 \%$ ) and mandibular (11 cases, $47.8 \%$ ) bones. In addition to these typical epithelial plaques, irregular- (Fig. $3 \mathrm{c}$ ) and round-shaped (Fig. 3d) epithelial islands were occasionally found ( 8 cases, $34.8 \%$ ). Sebaceous metaplasia of the epithelial islands was rarely observed (1 case, $4.3 \%)$. Mucous cells in partial epithelium were rarely found (3 cases, $13 \%$ ). To characterize the lining epithelium of LPC, immunohistochemistry for keratin species was examined. The lining epithelial cells, including epithelial plaques, were not positive for CK10 (Fig. 3e), but the whole layer of the lining was positive for CK17 (Fig. 3f), CK13 (not shown), and CK5/6 (not shown). Such immunohistochemical profiles suggested that the lining epithelium was of odontogenic origin.

\section{Epithelial plaques}

The size of epithelial plaques was determined on H-E stained sections as $0.50 \pm 0.13 \mathrm{~mm}$ in width and $0.13 \pm 0.04$ $\mathrm{mm}$ in height. Different from flat shaped lining epithelia, the characteristic epithelial plaques showed their own specific immunohistochemical features. In epithelial plaques, most surface cells and basal cells resemble each other not only in
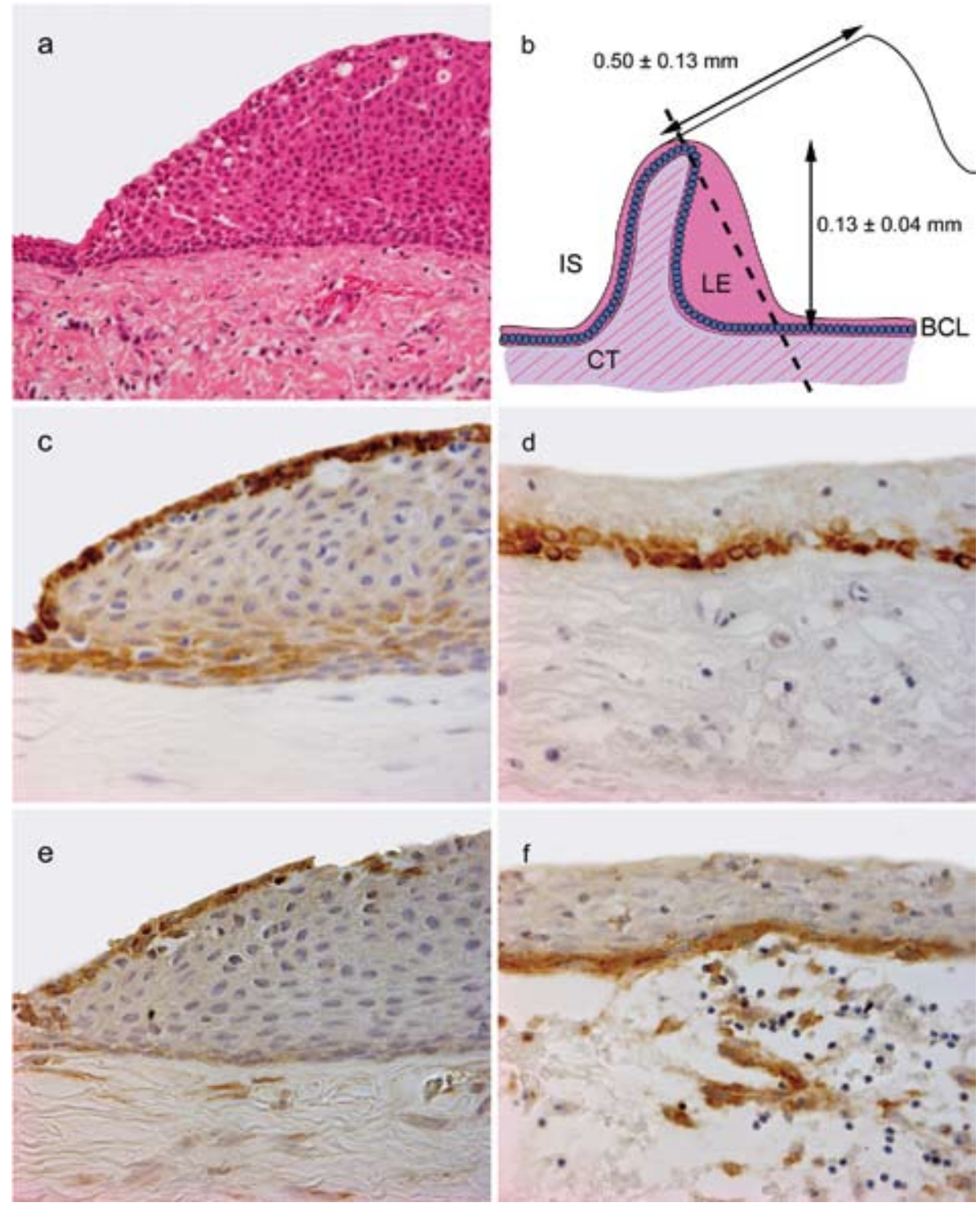

Fig. 4. Immunohistochemical architecture of epithelial plaques. (a, c, e) epithelial plaque, (b) schematic image of epithelial plaque, (d, f) flat epithelial lining. (a) HE stain; (c, d) immunoperoxidase stain for CK19, hematoxylin counterstain; (e, f) immunoperoxidase stain for integrin $\beta 1$, hematoxylin counterstain. (a) $\times 120$, (c-f) $\times 200$. IS: intracystic space; LE: lining epithelium; BCL: basal cell layer; CT: connective tissue; dotted line: cross sectioning position. In typical epithelial plaques (a), the most superficial surface epithelial cells were cuboidal in shape and resembled basal cells. Immunohistochemically, the most superficial surface cells were strongly positive for CK19 (c) and integrin $\beta 1$ (e), which were also positive in the basal and parabasal cells at the same time. In contrast, only basal cells were positive for CK19 (d) and integrin $\beta 1$ (f) in the flat epithelium $(\mathrm{d}, \mathrm{f})$. Based on H-E stain findings, the size of epithelial plaques was determined as $0.50 \pm 0.13 \mathrm{~mm}$ in width and $0.13 \pm 0.04 \mathrm{~mm}$ in height. Since the surface cells seemed to have basal cell-like immunohistochemical phenotypes, it was suggested that the epithelial plaques were generated by crosscutting of wrinkles or wavy protrusion of the lining epithelium as indicated by the dotted line (b). 
their cuboidal shapes but also in their immunohistochemical positivities for cytokeratin 19 (CK19) (Fig.4c) and integrin $\beta 1$ (Fig.4e), although CK19 (Fig. 4d) and integrin $\beta 1$ (Fig. 4f) were apt to be immunopositive only in the basal cells in flat shaped lining epithelium. The findings suggested that the uppermost cells had basal-cell-like characters, and therefore that the epithelial plaques were generated by cross-cutting of some wrinkle or wavy protrusions of the inner surface of LPC walls as schematically shown in Fig. $4 \mathrm{~b}$.

\section{Immunohistochemistry for differential diagnosis}

The characteristic epithelial plaques were the most important histological evidence for the differential diagnosis of LPC. When the lining epithelium lacked epithelial plaques, it was not always easy to make the diagnosis of LPC. Based on the findings in the cases with the characteristic epithelial plaques, immunohistochemical phenotypes of the lining epithelium of LPC were determined as shown in Fig. 5. When compared with keratocystic odontogenic tumor and radicular cyst, the LPC phenotypes were contrastively demonstrated. Ki-67-positive (+) cells were not found at all in LPC (Fig. 5d), while there were many Ki-67+ cells in the lining, especially in the parabasal cells, of keratocystic odontogenic tumor (Fig. 5e). As to the radicular cyst linings, $\mathrm{Ki}-67+$ cells were often found in the parabasal layer but were only sporadic in the basal layer (Fig. 5f). Podoplanin was only faintly immunolocalized in LPCs, while it was strongly positive in the basal cells of both keratocystic odontogenic tumor (Fig. 5h) and radicular cyst (Fig. 5i).

\section{Discussion}

The present study confirmed that the presence of the epithelial plaques, which was first referred to by Gold and Sliwkowski in 1973 (2), was definitely characteristic of the cystic lining of LPC, because they were found in all of the LPC cases examined. The pathogenesis of the characteristic epithelial plaque has never been discussed in past literature to the best of our knowledge. In the present study, we proposed for the first time the histogenetic process of the
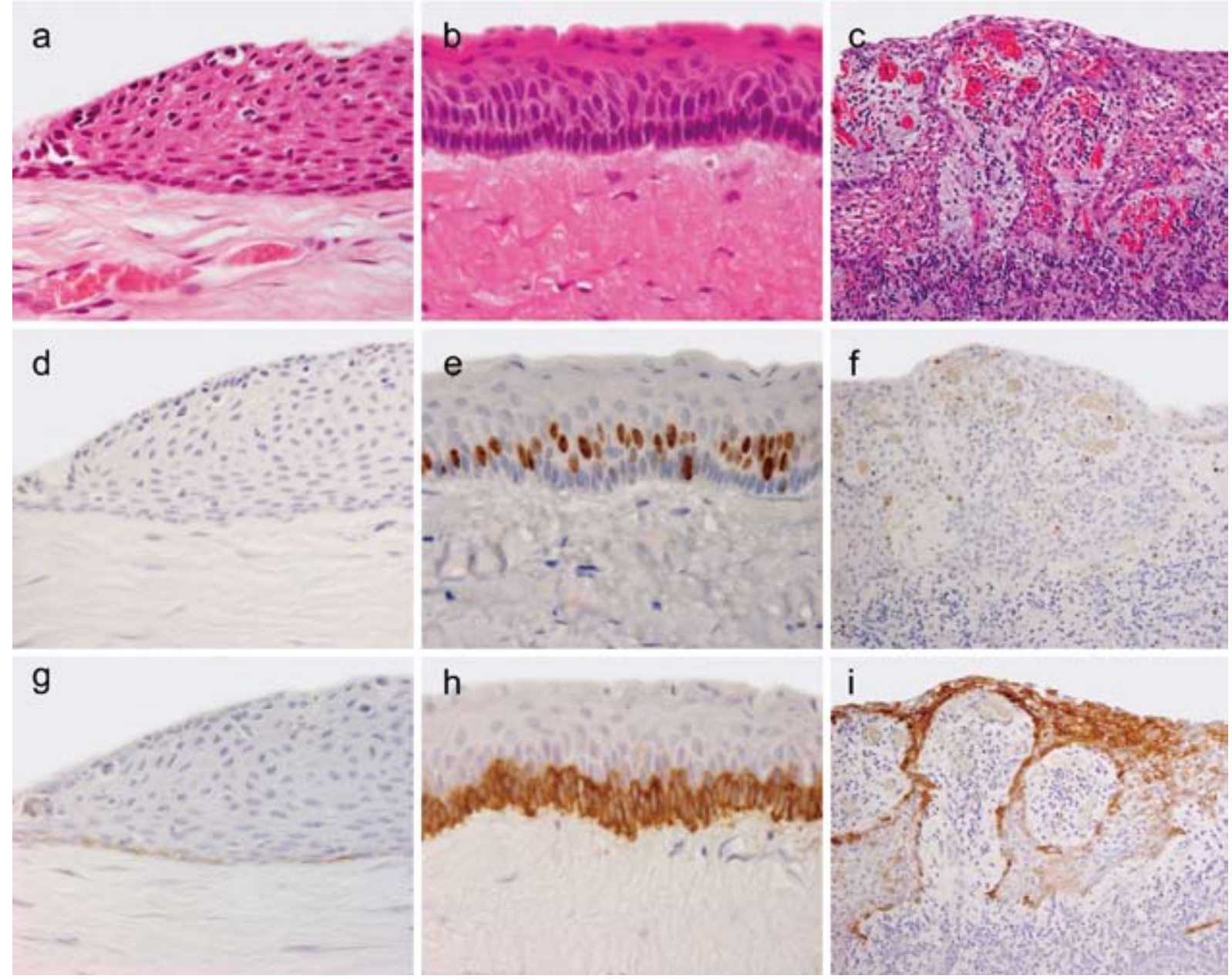

Fig. 5. Comparative immunohistochemistry between lateral periodontal cyst, keratocystic odontogenic tumor, and radicular cyst. (a, d, g) Lateral periodontal cyst; (b, e, h) keratocystic odontogenic tumor; (c, f, i) radicular cyst. (a-c) HE stain; (d-f) immunoperoxidase stain for Ki-67; (g-i) immunoperoxidase stain for podoplanin (D2-40), hematoxylin counterstain. $(\mathrm{a}, \mathrm{d}, \mathrm{g}) \times 120 ;(\mathrm{b}, \mathrm{e}, \mathrm{h}): \times 150 ;(\mathrm{c}, \mathrm{f}, \mathrm{i}) \times 70$. Ki-67 positive cells were not seen in LPC. Podoplanin positive cells were found in the basal layer of LPC linings. In contrast, both of them were highly enhanced in keratocystic odontogenic tumor, in which Ki-67 in parabasal cells and podoplanin were positive in basal cells. Lining epithelia of radicular cysts showed sporadic Ki-67 positivity in the basal layer and extensive podoplanin positivity from the basal to upper layers. 
epithelial plaque formation according to our three dimensional and speculative reconstructions of wavy protrusive wrinkles of the inner surface of the cyst wall. In addition, we were successful in characterizing the immunohistochemical features of the LPC lining epithelium, which might be useful for its differential diagnosis from other cystic jaw lesions, such as keratocystic odontogenic tumors or radicular cysts.

The mean age of 46 years in our present LPC series was slightly lower than the data reported from western countries, in which ages of $50(3,4)$ to $55(5)$ years were documented, indicating that LPC is a disease occurring or found in middle age. The most frequently reported locations of LPC were the mandibular premolar and molar areas (102 out of 174, a total number of documented cases, 58.6\%), followed by the maxillary anterior and premolar regions (45/174, 25.9\%) (3-11). Our series showed basically a similar tendency to those reports, although most of the maxillary cases (11/12, $91.7 \%$ ) were only found in the incisor region, and the mandibular ones were in both premolar $(5 / 11,45.5 \%)$ and molar regions $(6 / 11,54.5 \%)$ with an almost equal ratio. Since the tooth root involvement in the LPC lumina was mainly found in the maxillary cases, LPC, which is basically independent from teeth, might tend to grow larger into complicated shapes within the maxillary bone to eventually come in contact with tooth roots. In our present study, it was obvious that the size of LPCs was larger in the maxilla than in the mandible. The average size of LPCs in our series was larger than most of the reported cases, which were less than $1 \mathrm{~cm}$ in diameter (3-5, 8-10).

Clinical diagnoses of LPC were obtained in only $4.3 \%$ of the present series, indicating that its precise diagnosis before surgery is difficult. This is partly because LPC is symptomless and found by chance in radiographic examinations for other purposes $(2-3,6,8-9,11)$, and its typical X-ray findings are not always obtained (11).

The histopathogenesis of LPC is still poorly understood. Three possible epithelial tissues have been speculated as its origin: the reduced enamel epithelium of an erupting tooth, the rest of the dental lamina, and Malassez's epithelial cell rests (10). To characterize the lining epithelial cells of LPCs, we performed immunohistochemistry for keratin species in this study. In the present study, CK17, CK13, and CK5/6 were positive in the lining epithelium of LPC, whereas CK10 was not. CK13, an acidic type keratin localized in suprabasal layers of non-keratinized stratified epithelia has been shown to be expressed in the lining epithelia of most odontogenic cysts (13-15). CK5/6, a neutral-basic type of keratin, was also localized in the lining epithelia of most odontogenic cystic lesions (14). CK17, an acidic type keratin, has been regarded as one of the most representative keratin species expressed in developmental odontogenic lesions, because its expression was most enhanced in keratocystic odontogenic tumors, followed by dentigerous cysts (13-15). CK10, an intermediate-sized acidic type keratin, has been immunolocalized in keratocystic odontogenic tumors as well as in dentigerous cysts but not in radicular cysts (14). Based on these findings, the present immunohistochemical results on the LPC lining epithelia, which were immunopositive for CK5/6, CK13 and CK17, indicate that LPC is of odontogenic origin. Since there was no CK10 positivity in LPC, the immunohistochemistry for CK10 may be useful in the differential diagnosis of LPC from keratocystic odontogenic tumor or dentigerous cyst.

No histopathogenesis of the characteristic epithelial plaques in the LPC lining has been discussed in the literature, although their presence in LPC has been widely referred to (2-11). In the present observation, we demonstrated for the first time that the uppermost cells of the epithelial plaque had some basal cell characteristics based on their immunohistochemical positivities for CK19 and integrin $\beta 1$, which were usually found in the basal cells of the oral squamous epithelia (15-18). This finding might be important for speculating how epithelial plaque is generated. As shown in the scheme of Fig. 4b, cross and oblique cutting of wrinklelike structures in the inner surface may be only one possibility for generation of such a peculiar structure.

Immunohistochemically, there were scarce numbers of Ki-67-positive cells in the lining epithelium of LPCs. This finding confirms that the cell proliferation activity in the LPC linings is very low and completely different from that of keratocystic odontogenic tumor (19-20), and also that LPC is characterized as a slow-developing cyst found in the later life period. Therefore, Ki-67 immunohistochemistry is useful in the differential diagnosis of LPC from other cystic jaw lesions, especially keratocystic odontogenic tumor. In addition, no obvious podoplanin immunopositivity was shown to be useful in the distinction of LPC from other jaw cysts.

The histopathological criteria of LPC have been particularly clarified in the present study. However, its pathogenesis arising between tooth roots is still unclear. Recently, keratocystic odontogenic tumor has been well established as a neoplastic disease and has been separated from the rather obscure entity of primordial cyst. Then, what sorts of cysts are left in the previous (old or classic) concept of primordial cysts which lack keratinization of their linings, after keratocystic odontogenic tumor has been excluded from them? One possibility we could propose at this point is that some of the LPCs were considered or diagnosed as primordial cysts and that primordial cyst never existed, although further investigations are needed to resolve the controversy.

\section{References}

1. Mezrow RR. Case report of a paradental cyst. J Am Dent Assoc $1950 ;$ 41: 77-8.

2. Gold L, Sliwkowski AS. Lateral periodontal cyst: a clinical and histological study. Trans Int Conf Oral Surg 1973; 4: 85-9.

3. Altini M, Shear M. The lateral periodontal cyst: an update. J Oral Pathol Med 1992; 21: 245-50.

4. Wysocki GP, Brannon RB, GardnerDG, Sapp P. Histogenesis of the lateral periodontal cyst and the gingival cyst of the adult. Oral Surg Oral Med Oral Pathol 1980; 50: 327-34.

5. Rasmusson LG, Magnusson BC, Borrman H. The lateral 
periodontal cyst. A histopathological and radiographic study of 32 cases. Br J Oral Maxillofac Surg 1991; 29: 54-7.

6. Fantasia JE. Lateral periodontal cyst. An analysis of 46 cases. Oral Surg Oral Med Oral Pathol 1979; 48: 237-43.

7. Cohen DA, Neville BW, Damm DD, White DK. The lateral periodontal cyst. A report of 37 cases. J Periodontol 1984; 55: $230-4$

8. Kerezoudis NP, Donta-Bakoyianni C, Siskos G. The lateral periodontal cyst: aetiology, clinical significance and diagnosis. Endod Dent Traumatol 2000; 16: 144-50.

9. Saygun I, Ozdemir A, Safali M. Lateral periodontal cyst. Turk J Med Sci 2001; 31: 375-8.

10. Angelopoulou E, Angelopoulos AP. Lateral periodontal cyst. Review of the literature and report of cases. $J$ Periodontol 1990; 61: 126-31.

11. Mendes RA, van der Waal I. An unusual clinicoradiographic presentation of a lateral periodontal cyst-report of two cases. Med Oral Patol Oral Cir Bucal 2006; 11: E185-7.

12. Kramer IRH, Pindborg JJ, Shear M. Histological Typing of Odontogenic Tumours, Second Edition, World Health Organization International Histological Classification of Tumours. Springer-Verlag, Berlin, 1992; 37, 107-8.

13. Meara JG, Pilch BZ, Shah SS, Cunningham MJ. Cytokeratin expression in the odontogenic keratocyst. J Oral Maxillofac Surg 2000; 58: 862-5.
14. Stoll C, Stollenwerk C, Riediger D, Mittermayer C, Alfer J. Cytokeratin expression patterns for distinction of odontogenic keratocysts from dentigerous and radicular cysts. $J$ Oral Pathol Med 2005; 34: 558-64.

15. Hormia M, Ylipaavalniemi P, Nagle RB, Virtanen I. Expression of cytokeratins in odontogenic jaw cysts: monoclonal antibodies reveal distinct variation between different cyst types. J Oral Pathol Med 1987; 16: 338-46.

16. Sawaf MH, Ouhayoun JP, Shabana AH, Forest N. Cytokeratin expression in human tongue epithelium. Am J Anat 1990; 189: 155-66.

17. Farmer I, Freysdottir J, Dalghous AM, Fortune F. Expression of adhesion and activation molecules in human buccal epithelial cell lines and normal human buccal epithelium in situ. J Oral Pathol Med 2001; 30: 113-20.

18. Chainani-Wu N, Lagenaur LA, Penaranda ME, Palefsky JM. Integrin expression in oral hairy leukoplakia and normal tongue epithelium. Oral Dis 2000; 6: 234-40.

19. Kichi E, Enokiya Y, Muramatsu T, et al. Cell proliferation, apoptosis and apoptosis-related factors in odontogenic keratocysts and in dentigerous cysts. J Oral Pathol Med 2005; 34: 280-6.

20. Tsuneki M, Cheng J, Maruyama S, Ida-Yonemochi H, Nakajima M, Saku T. Perlecan-rich epithelial linings as a background of proliferative potentials of keratocystic odontogenic tumor. J Oral Pathol Med 2008; 37: 287-93. 